\title{
A Case Demonstrating the Ribaroxaban Therapy for Paradoxical Embolism
}

\author{
Hiroto Aikawa ${ }^{\text {a, b }}$, Kazuhiro Shimizu ${ }^{\text {a, c }}$, Mahito Noro ${ }^{a}$
}

\begin{abstract}
A paradoxical embolism is defined as a systemic arterial embolus due to passage of a venous thrombus through a right to left shunt. In recent years, vitamin $\mathrm{K}$ antagonists and aspirin are used as anticoagulant medications for the secondary prevention of paradoxical embolism. We describe a case of subacute right upper limb ischemia due to paradoxical embolism. We first started treatment with urokinase, intravenous (IV) injection and unfractionated heparin (continuous IV). As her condition and the serum D-dimer level showed improvement, we started catheterization on day 7 after admission; however the right brachial artery thrombus did not disappear. For her outpatient care, based on the viewpoint of providing rapid anticoagulation therapy within the therapeutic range, having longest periods of initial intensive therapy we chose the treatment using ribaroxaban. Recanalization of her right brachial artery was achieved with this therapy. Here, we report the effective results of initial intensive therapy using ribaroxaban for paradoxical embolism.
\end{abstract}

Keywords: Paradoxical embolism; Ribaroxaban; PFO; VTE; DOACs

\section{Introduction}

Patent foramen ovale (PFO) is one of the most important causes of paradoxical embolism, involving the passage of a thrombus from the venous circulation to the systemic arterial circulation through a right to left shunt [1]. The diagnostic criteria of paradoxical embolism are the following conditions: 1) No lesion becomes the embolus source in systemic arterial circulation; 2) Existence of the deep vein thrombosis (DVT) or the pulmonary embolism becoming the embolus source; 3) Existence of right to left shunt; 4) A favorable pressure gradient must exist at some time in the cardiac cycle to promote right-

Manuscript submitted January 30, 2019, accepted February 7, 2019

aDepartment of Internal Medicine, Toho University Sakura Medical Center, Chiba, Japan

${ }^{b}$ Department of Internal Medicine, Toho University Omori Medical Center, Tokyo, Japan

cCorresponding Author: Kazuhiro Shimizu, Department of Internal Medicine,

Toho University Sakura Medical Center, Chiba, Japan.

Email: k432@sakura.med.toho-u.ac.jp

doi: https://doi.org/10.14740/cr840 to-left shunting [2-4]. PFO is found in about $15-27 \%$ of the population [5]. The final resting place of the thrombus is common in the brain when it becomes clinically identifiable. The artery embolism in paradoxical embolism is said to be formed in the brain in $37 \%$ cases, primarily peripheral $49 \%$ and so on [6]. Furthermore, it is reported that cerebral infarction develops in more than 200,000 patients per year in Japan, and more than $5 \%$ are related to PFO [7]. Anticoagulant therapy is effective for the prevention of the DVT [8-10], which causes the paradoxical embolism. We report the effectiveness of initial intensive therapy using ribaroxaban in a case of paradoxical embolism.

\section{Case Report}

A 67-year-old woman presented to our hospital with a 2-day history of pain and a feeling of coldness in her right hand. In her past medical history she had not received any treatment for dyslipidemia. There is no other medical history or smoking history. Upon physical examination she had no heart murmur or leg edema; her blood pressure was 154/96 mm Hg (left brachial), and oxygen saturation of peripheral artery was $96 \%$ at room air. Her right hand was pale and the right radial artery was pulseless. An electrocardiogram (ECG) showed sinus rhythm at $79 \mathrm{bpm}$. The serum D-dimer level was slightly increased $(2.18 \mu \mathrm{g} / \mathrm{mL})$. Computed tomography (CT) scan confirmed thromboembolism in the distal part of the right brachial artery, left pulmonary artery and right kidney infraction (Fig. 1). Cerebral infraction and stenosis of the main cerebral artery were not detected by the head magnetic resonance imaging (MRI)/magnetic resonance angiography (MRA).

Doppler sonography of right upper limb revealed the disappearance of blood flow at the distal part of the brachial artery, and collateral circulation to the radial artery from the brachial artery. In this case, multiple thromboemboli occurred in both the systemic and pulmonary circulation. Thus, we suspected paradoxical thromboembolism. Transesophageal echocardiogram and venous ultrasonography findings showed the presence of a PFO and DVT (Fig. 2). Thus, we diagnosed paradoxical embolism due to PFO. Photoplethysmogram (PTG) of her right hand was very slow on admission (Fig. 3). The condition of her right upper limb had been relieved from 2 days previously due to the development of collateral circulation. Therefore, we thought that neither emergency operation nor catheter intervention for thrombectomy was necessary in this patient. We started treatment with urokinase (UK) 240,000 U/day in- 


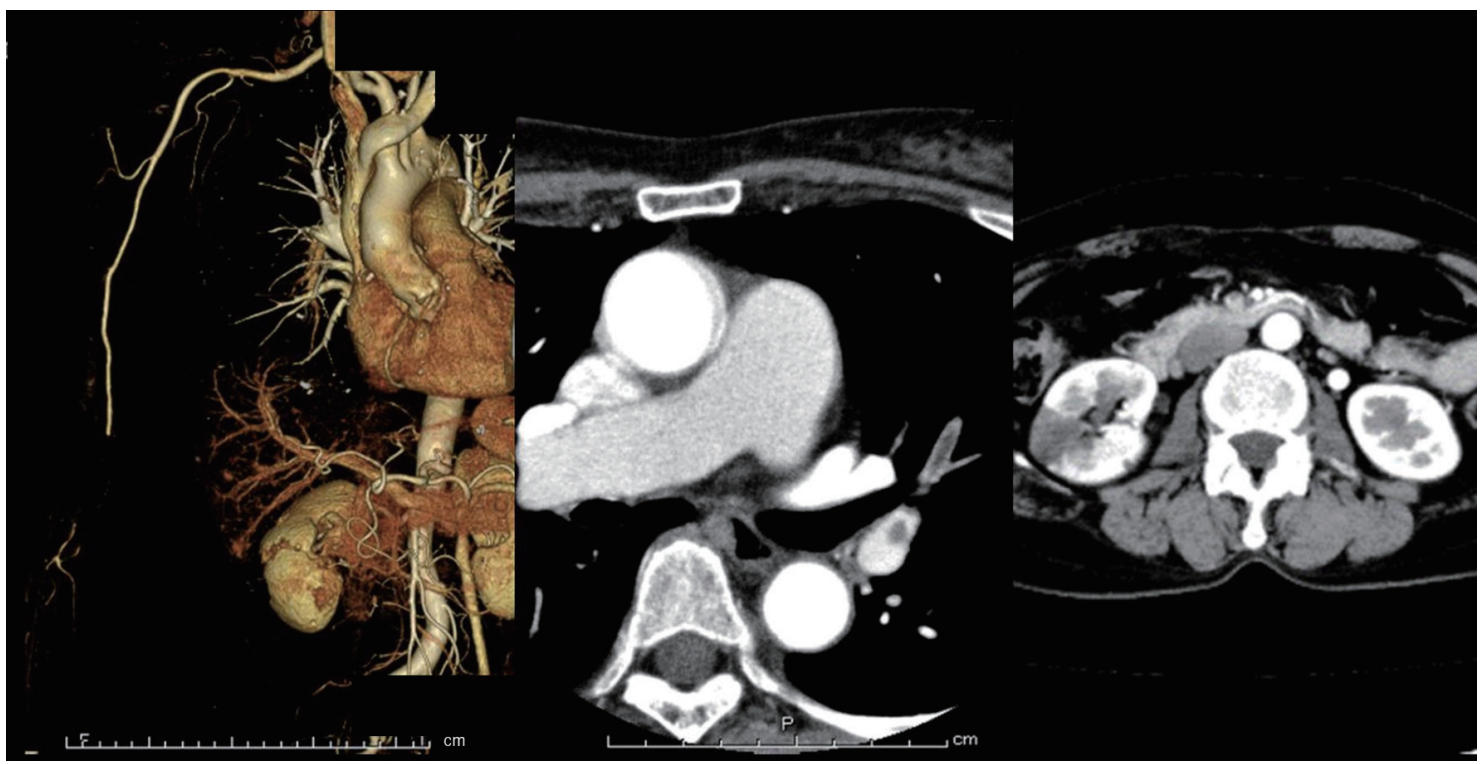

Figure 1. Enhanced computed tomography of the patient. 3D-CT scan shows interruption of the blood flow in the distal part of brachial artery and development of collaterals circulation (left photo). Contrast CT scan shows left pulmonary artery thromboembolism (middle photo) and right kidney infraction (right photo). CT: computed tomography.

travenous injection (IV) and unfractionated heparin (UFH) continuous IV (target activated partial thromboplastin time (APTT) 60 - $80 \mathrm{~s}$ ). As her condition and the serum D-dimer level were improving we started catheterization at day 7 after admission (Fig. 4); however the thrombus still remained in the brachial artery. Although her pain was relieved, her right hand was still cold and PTG of the right hand at day 7 was slow. For her outpatient care, based on the viewpoint of providing rapid anticoagulant therapy within the therapeutic range, having longest periods of initial intensive therapy we chose treatment using ribaroxaban (15 mg, two tablets twice daily) (Fig. 5). Three weeks after discharge from the hospital, her right hand had become warm and the serum D-dimer level and PTG were normalized (Fig. 3). From that time, we changed ribaroxaban to the maintenance dose (15mg, one tablet once daily). Three months after discharge, Doppler sonography and MRA of her right arm confirmed the achievement of reperfusion of her brachial artery (Fig. 4).

\section{Discussion}

Paradoxical embolism occurs when right atrium pressure exceeds left atrium pressure. The rise of right atrium pressure

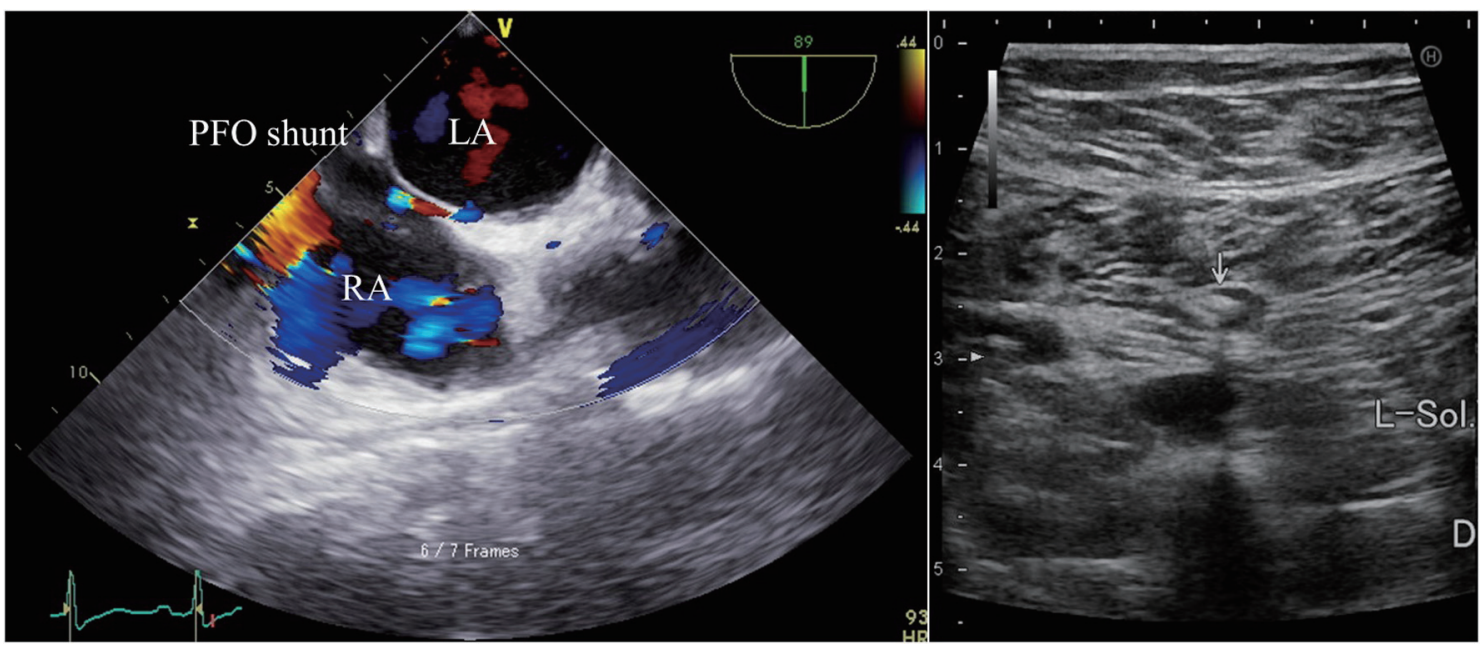

Figure 2. The imaging of transesophageal echocardiography and venous ultrasonography. Transesophageal echocardiography (left photo) shows the presence of patent foramen ovale (PFO) and right to left shunt in Valsalva maneuver. Interatrial septal aneurysm and left atrial thrombus are not observed. Venous ultrasonography (right photo) shows presence of deep vein thrombosis in left soleal vein. LA: left atrium; RA: right atrium. 


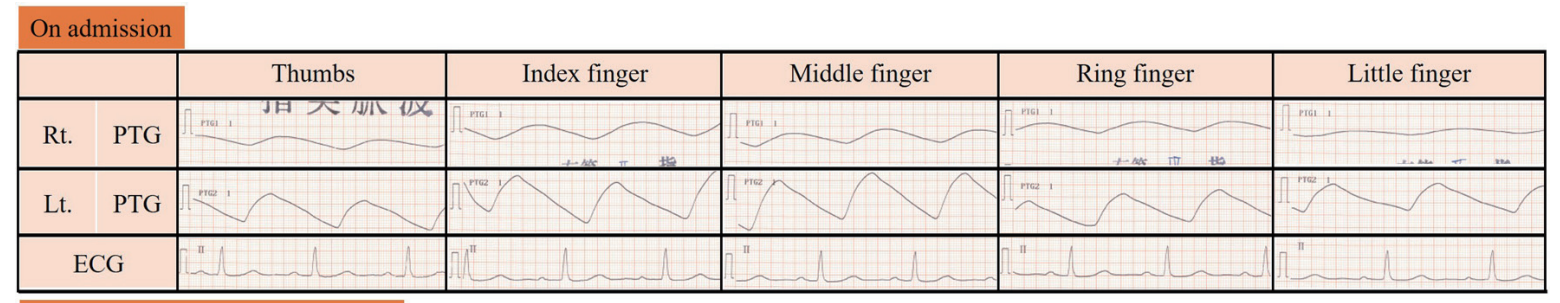

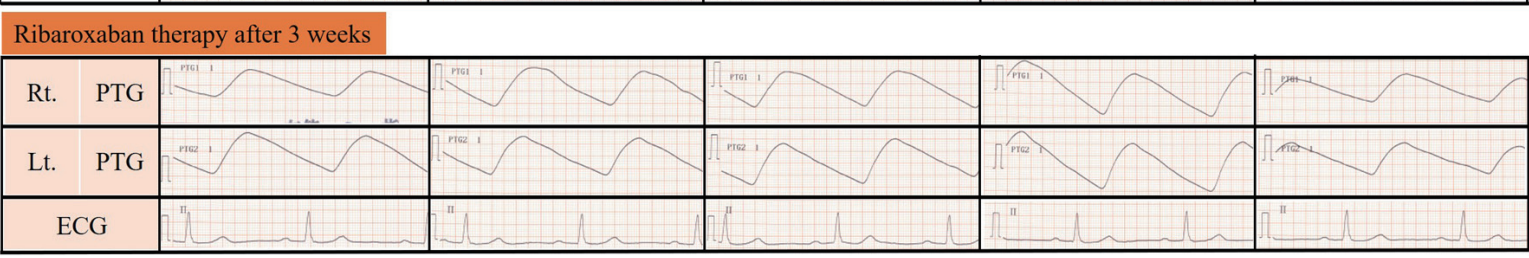

Figure 3. The imaging of photoplethysmogram. The figure shows photoplethysmogram (PTG) of patient fingers. Upper section is her right hand, lower section is her left hand, finger number from right to left. On admission, her PTG was slow. After ribaroxaban therapy for 3 weeks, her PTG was normalized. PTG: photoplethysmogram; ECG: electrocardiogram.

is caused by obstruction of pulmonary blood flow by pulmonary embolus, increased transmural pressures such as transient cough or Valsalva strain, right ventricular hypokinesis and so on [8]. In the present case, we considered acute artery obstruction caused by arteriosclerosis as a differential diagnosis, but there was no significant atherosclerosis such as stenosis or intravascular plaque in her medical image findings. Although the UK and UFH therapies were ineffective at acute phase, the initial intensive therapy with ribaroxaban could achieve thromboembolism regression without any significant side effects. UK and UFH therapy could not achieve thrombus regression at acute phase. The cause of thrombus was not resolved possibly due to the short treatment period, because the serum D-dimer level was improved. But it was beneficial that she could receive effective and safe outpatient care with ribaroxaban therapy. An anticoagulant therapy is also effective for the recurrence prevention of the venous thromboembolism (VTE) which can become the embolism source of the paradoxical cerebral embolism. Factor Xa inhibitors included in direct oral anticoagulants (DOACs) are potent, oral direct inhibitors of prothrombinase-bound, clot-associated or free FXa. These agents have a rapid onset of action, a wide therapeutic window, little or no interaction with food and other drugs, minimal inter-patient variability, and display similar pharmacokinetics in different patient populations. But the precise mechanism by which the factor Xa inhibitor causes thrombus regression remains unclear, and no fibrinolytic activity of this agent has been described. Initiation of fibrinolysis occurs through a number of orchestrated interactions among fibrin and plasminogen as well as its activator and results in the generation of plasmin [9]. Therefore, the efficacy of ribaroxaban may reflect the potency of the fibrinolytic response relative to that of the coagulation response [10]. Factor Xa inhibitors provide numerous benefits over older vitamin $\mathrm{K}$ antagonists (VKAs) due to major pharmacological differences. VKAs are economical and very well characterized, but have important limitations that can outweigh these advantages, such as slow onset of action, narrow therapeutic window and unpredictable anticoagulant effect

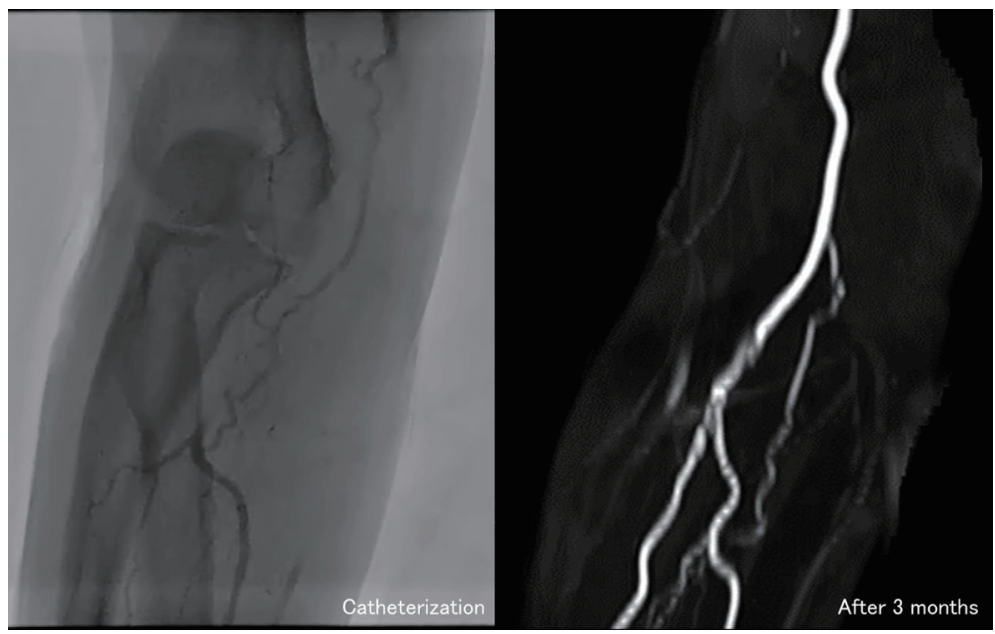

Figure 4. The imaging of brachial artery angiography. Catheterization shows the thrombus still remains in brachial artery and development of collateral circulation on the seventh day after admission (left photo). Three months after discharge, MRA shows brachial artery could achieve reperfusion (right photo). MRA: magnetic resonance angiography. 


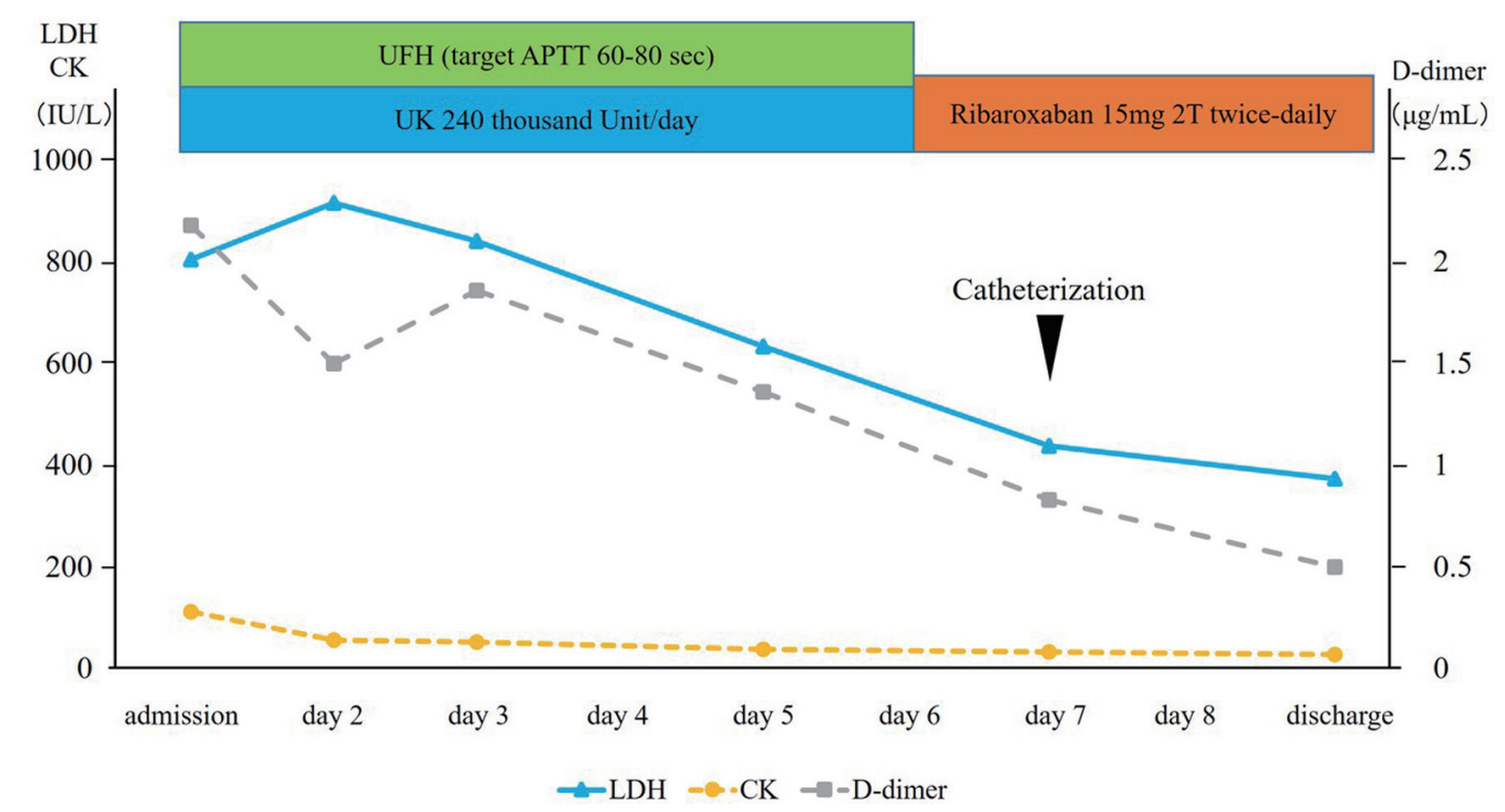

Figure 5. Clinical course of the patient. UFH: unfractionated heparin; UK: urokinase; APTT: activated partial thromboplastin time; LDH: lactate dehydrogenase; CK: creatine kinase.

[11]. Recent clinical trials have shown the efficacy and safety of VTE using DOACs [12-16]. DOACs will be the first choice for paradoxical embolism medical therapy in the future with VKA and aspirin. In the present case, the transcatheter PFO closure (PFO closure) as the secondary prevention of cerebral infraction is specified as class IIb in the AHA/ASA guidelines 2014. PFO closure was first reported in 1992. Subsequently, various studies of PFO closure were reported. Shah et al reported that in patients with PFO and cryptogenic stroke, transcatheter device closure decreases the risk for recurrent stroke compared with medical therapy alone; however PFO closure might affect atrial fibrillation [17], and it may cause some procedure-related complications. In the near future, an optimal strategy for preventing recurrent stroke in patients with cryptogenic stroke and PFO will be designed.

We reported the effective initial intensive therapy using ribaroxaban for paradoxical embolism. DOACs will be the reliable choice for medical therapy of paradoxical embolism.

\section{Acknowledgments}

We deeply appreciate the advice of Professor Takanori Ikeda.

\section{Funding Disclosure}

None.

\section{Conflict of Interest}

The authors have declared no conflicts of interest.

\section{Ethical Statement}

All procedures followed were in accordance with the ethical standards of the responsible committee on human experimentation (institutional and national) and with the Helsinki Declaration of 1964 and later versions.

\section{Informed Consent}

Informed consent was obtained from the patient for being included in this report.

\section{References}

1. Mirarchi FL, Hecker J, Kramer CM. Pulmonary embolism complicated by patent foramen ovale and paradoxical embolization. J Emerg Med. 2000;19(1):27-30.

2. Meister SG, Grossman W, Dexter L, Dalen JE. Paradoxical embolism. Diagnosis during life. Am J Med. 1972;53(3):292-298.

3. Aburahma AF. Work-up and management of patients with paradoxical emboli. W V Med J. 1996;92(5):260-264.

4. AbuRahma AF, Downham L. The role of paradoxical arterial emboli of the extremities. Am J Surg. 1996;172(2):214-217.

5. Hagen PT, Scholz DG, Edwards WD. Incidence and size of patent foramen ovale during the first 10 decades of life: an autopsy study of 965 normal hearts. Mayo Clin Proc. 1984;59(1):17-20.

6. Loscalzo J. Paradoxical embolism: clinical presentation, diagnostic strategies, and therapeutic options. Am 
Heart J. 1986;112(1):141-145.

7. Ueno Y, Iguchi Y, Inoue T, Shibazaki K, Urabe T, Kimura K. Paradoxical brain embolism may not be uncommonprospective study in acute ischemic stroke. J Neurol. 2007;254(6):763-766.

8. Meier B, Lock JE. Contemporary management of patent foramen ovale. Circulation. 2003;107(1):5-9.

9. Medved L, Nieuwenhuizen W. Molecular mechanisms of initiation of fibrinolysis by fibrin. Thromb Haemost. 2003;89(3):409-419.

10. Morishima Y, Kamisato C, Honda Y. Treatment of venous thrombosis with an oral direct factor $\mathrm{Xa}$ inhibitor edoxaban by single and multiple administrations in rats. Eur J Pharmacol. 2014;742:15-21.

11. Scaglione F. New oral anticoagulants: comparative pharmacology with vitamin $\mathrm{K}$ antagonists. Clin Pharmacokinet. 2013;52(2):69-82.

12. Gomez-Outes A, Suarez-Gea ML, Lecumberri R, Terleira-Fernandez AI, Vargas-Castrillon E. Direct oral anticoagulants in the treatment of venous thromboembolism, with a focus on patients with pulmonary embolism: an evidence-based review. Vasc Health Risk Manag. 2014;10:627-639.

13. Schulman S, Kakkar AK, Goldhaber SZ, Schellong S,
Eriksson H, Mismetti P, Christiansen AV, et al. Treatment of acute venous thromboembolism with dabigatran or warfarin and pooled analysis. Circulation. 2014;129(7):764772.

14. Schulman S, Wahlander K, Lundstrom T, Clason SB, Eriksson H, Investigators TI. Secondary prevention of venous thromboembolism with the oral direct thrombin inhibitor ximelagatran. N Engl J Med. 2003;349(18):17131721.

15. EINSTEIN Investigators, Bauersachs R, Berkowitz SD, Brenner B, Buller HR, Decousus H, Gallus AS, et al. Oral rivaroxaban for symptomatic venous thromboembolism. N Engl J Med. 2010;363(26):2499-2510.

16. Shimizu K, Iiduka T, Sato S, Kiyokawa H, Nakagami T, Mikamo H, Kawazoe M, et al. The clinical course of symptomatic deep vein thrombosis after 3 months of anticoagulant therapy using fondaparinux/edoxaban or fondaparinux/vitamin K antagonist. Ther Clin Risk Manag. 2018;14:377-383.

17. Shah R, Nayyar M, Jovin IS, Rashid A, Bondy BR, Fan TM, Flaherty MP, et al. Device closure versus medical therapy alone for patent foramen ovale in patients with cryptogenic stroke: a systematic review and meta-analysis. Ann Intern Med. 2018;168(5):335-342. 